\title{
A Proposal for Elastic Spectrum Management in Wireless Local Area Networks
}

\author{
Luis Amaral Lopes (luis.amaral@ulusofona.pt), Rute Sofia (rute.sofia@ulusofona.pt), Hassan Osman \\ (H.Osman@kent.ac.uk), Huseyin Haci (hh219@kent.ac.uk)
}

This is the author's pre-print version. Personal use of this material is permitted. However, permission to reprint/republish this material for advertising or promotion or for creating new collective works for resale or for redistribution to thirds must be obtained from the copyright owner. The camera-ready version of this work has been published at INFOCOM'2014 Demo/Poster Session, date of April 2014 and is property of IEEE.

\begin{abstract}
Wireless networks represent today the last hop to the Internet end-user, thus used to interconnect multiple users, that should ideally be served simultaneously. Still, its technical design implies that only one user can be served per unit of time, design aspect which limit the potential applicability of wireless in dense environments. This technological demonstration shows a novel wireless extension mechanism, backward compatible with the current standards, that is capable of providing downstream transmission to multiple users within the same time-frame. The demonstration is based on a software defined wireless framework derived from the UNIX module mac80211.
\end{abstract}

\section{INTRODUCTION}

User-centric networks (UCNs) reflect a recent architectural trend of self-organizing, autonomic networks where the Internet end-user cooperates by sharing network services and resources. UCNs are characterized by spontaneous and grassroots deployments of wireless architectures (e.g. ad-hoc or infrastructure), where users on such environments roam frequently and are also owners of networking equipment (e.g. residential Access Points) thus playing an active role in terms of topology changes (e.g. APs that dynamically become active or inactive).

In environments such as the ones described, the current wireless technologies (IEEE 802.11) fall short in being able of serving multiple users during the same duty cycle of a AP, as the way IEEE 802.11 was devised only allows communication to a device in a specific duty cycle of an AP.

Within the context of the European project User-centric Wireless Local Loop (ULOOP) [2] resource management has been addressed to allow more fairness in the scenarios described, in a way that is agnostic to the end-user. One of the aspects addressed relates with an extension applicable to IEEE 802.11, which is fully backward compatible with

Luis Lopes is with the COPELabs, University Lusófona, Lisbon, Portugal. Rute Sofia is with the COPELabs, University Lusófona, Lisbon, Portugal. Hassan Osman is with the University of Kent, Kent, United Kingdom. Huseyin Haci is with the University of Kent, Kent, United Kingdom.

978-1-4799-3360-0/14/\$31.00 (C)2014 IEEE the current set of IEEE 802.11 standards, and yet allows a controller to send data downstream to multiple stations, within the same duty cycle. Our approach is novel in the fact that considers a software defined approach only for the MAC Layer and completely transparent to others OSI layers, thus being applicable in current infrastructures without requiring any changes to the hardware, or even to current standards.

This extension of the MAC layer, which we have named Elastic Spectrum Management (ESM)[5], follows the recent trend concerning frequency assignment and sub-division which argues that the channel width of nodes should be adaptive. Taking this into account, we developed and implemented an alternative way of arranging wireless channel assignments, based on Orthogonal Frequency Division Multiplexing (OFDM), currently integrated in IEEE $802.11 \mathrm{a} / \mathrm{g} / \mathrm{n}$ standards.

\section{Elastic SPectrum Management}

ESM software architecture has been provided as a patch to the UNIX module mac80211, and has two modes of operation, node or gateway. Usually, the node mode is activated if ESM is installed in a station, while the gateway mode is activated if ESM is installed in a Wi-Fi access point.

When activated as gateway ESM controls the amount of OFDM sub-frequencies that a gateway can assign to multiple users downstream (from the controller to the station), as well as the way that the MAC layer controls the access to the configured sub-frequency chunks. The number of subfrequencies that a gateway can assign to a node depends on the node relevancy within a specific context, which in ULOOP is related to a trust level ${ }^{1}$ within a specific community [3].

For upstream communication (from the station to the controller), ESM follows the regular MAC operation, as defined by IEEE 802.11 standards. Then downstream, let us consider the example of IEEE 802.11g in Europe to better explain what ESM does, for the band of $2.4 \mathrm{GHz}$ usual in Europe. With IEEE $802.11 \mathrm{~g}$, each channel occupies $16.25 \mathrm{MHz}$ of bandwidth at the $2.4 \mathrm{GHz}$ frequency range. Then, each channel is divided into 52 sub-carriers of $312.5 \mathrm{kHz}$. Each sub-carrier can apply a specific modulation scheme, e.g. QPSK, 16-QAM. Out of the 52 sub-carriers, 4 of them are used for pilot proposes and 48 are used for data. Together, these sub-carriers overlap to fully utilize the $16.25 \mathrm{MHz}$ channel bandwidth dedicated per channel, for a concrete duty cycle.

\footnotetext{
${ }^{1}$ ESM has been implemented in an independent way and therefore, other parameters, instead of the trust notion, can be used to define a notion of fairness.
} 
What ESM adds in is that it allocates only a subset of subcarriers to each station. The subset is computed based on a specific prioritization. ESM is validated and compared with IEEE 802.11 standards in [4].

\section{A. Gateway Mode}

One controller is expected to serve multiple stations, here referred to as $s 1, \ldots$, sn. Each time a new station requests resources from a controller, it is assigned with a specific virtual queue (based on MAC layer virtualization techniques available today) which gets associated to the identifier of the station that is transmitted with the data frames of the station. The stations may then be served by their controller according to their priority in the system - the highest priority stations get served first and may get greater amount of resources than other low priority stations.

The controller periodically serves the queues assigned to each station, and creates the MAC frames to be sent takes care of creating a payload that holds sets of bits for several stations $\mathrm{s} 1, \ldots, \mathrm{sn}$. This is not a new MAC frame; instead, we simply consider a new way to interpret the MAC frame payload. Hence, stations that implement ESM interpret the modified payload, by extracting only the bits that are assigned to them; regular IEEE 802.11 stations will simply discard that content, following the regular MAC Layer procedure.

To be capable of providing such a payload, ESM relies on a specific bit-arrangement technique, which considers that for each OFDM symbol transmission, data bits from s1 to sn can be transmitted simultaneously. In IEEE 802.11g, the number of inputs to IFFT is $\mathrm{N}=48$. $\mathrm{N}$ is then split across the number of stations to serve in a duty cycle. For instance, if we consider two stations, e.g. s1 and s2, the controller applies, based on tokens of s1 and s2, a priority policy and allocates e.g. 12 inputs to s1 and 36 inputs to s2. The used technique also considers the modulation level, i.e. $\mathrm{M}$ bits per input, supported by the current transmission period. In total, the IFFT has $\mathrm{N}^{*} \mathrm{M}$ bits capacity per OFDM symbol. Providing an example for a modulation level $\mathrm{M}=4$ and the two stations s1 and s2, in each OFDM symbol transmission, a series of $48 * 4=192$ bits would be transmitted, where the first $12 * 4=48$ bits are directed to $\mathrm{s} 1$, and the rest $36 * 4=144$ bits go for station s2.

ESM is available as open-source software [1], licensed under LGPLv3.0.

\section{B. Node Mode}

When node mode is activated, the station checks each frame overheard and look for a specific flag at the MAC header, which identifies a modified ESM frame. If this is a normal station (not implementing ESM) the regular MAC procedure for unknown frames is considered. If it is an ESM frame, the station reads, from inside the received frame payload, the control bits searching for its own identifier. Data identified as belonging to other stations is discarded via the regular MAC operation. A specific data length parameter (provided as control bit) allows a station to ensure that it gets its own data block. Moreover, the last block is identified via an End of Data (EoD) flag. The station then puts all of the received pieces of data in a new MAC frame which is solely used to allow the MAC operation to proceed without changes.

\section{DEMO SCENARIO}

The demo, represented in Figure 1, consists of two WiFi (802.11b/g) Access Points and two laptops (stations) connected to the AP via a specific SSID, ULOOP_OPEN. The APs rely on the Atheros chipset AR7240, and both APs run OpenWRT Backfire 10.03.1. The AP identified as ULOOP has in addition ESM installed. The white laptop, represented in Figure 1, is a Toshiba Satellite L850-1P9, running Ubuntu 12.04 with kernel 3.5. The black laptop, is a Toshiba Satellite Pro R850-1JJ, running Ubuntu 11.10 with kernel 3.0. The demo shows the performance of ESM against IEEE 802.11, based on real streaming of videos, via the Internet. The demo is split into two phases: On a first phase we show how the two stations communicating via regular IEEE 802.11, and then show the ESM operation.

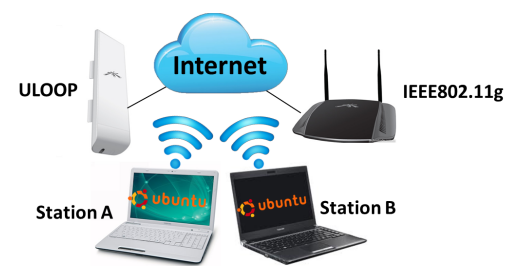

Figure 1. Demo configuration

The data rate is limited to $1 \mathrm{Mbps}$ to emulate the regular MAC broadcast operation, as this is how ESM works also. Then, two different videos (from Youtube or another Internet server) are started on the stations, showing the impact of MAC contention on real streaming, first for plain IEEE 802.11g, and then with ESM.

What will be visible is the shorter amount of buffering pauses, due to the downstream multi-user transmission. By reducing delays, ESM provides a better user experience.

\section{SUMMARY}

This demo provides a novel, backward compatible extension of MAC802.11 that improves wireless fairness based on downstream multi-user transmission. ESM provides benefits in the form of latency as well as improved fairness, an aspect that is essential to allow Wi-Fi to operate adequately in Internet access shared scenarios, of which UCNs are an example. The reduction in latency and the possibility to prioritize requests according to the subscriber priority introduces a way to prevent problems such as the "hidden station" problem. ESM is available as open-source software licensed under LGPLv3.0, and has been specified to ensure full compatibility with the current set of IEEE 802.11 standards.

\section{ACKNOWLEDGEMENTS}

This work has been developed under the EU IST FP7 project ULOOP - User-centric Wireless Local Loop - grant number 257418 . 


\section{REFERENCES}

[1] Available at http://uloop.eu/ uloop/software/RM.zip.

[2] European IST FP7 ULOOP - User-centric Wireless Local Loop, 20102013. Available at http://uloop.eu.

[3] C. Ballester, Jean-Marc Seigneur, Paolo Di Francesco, V. Moreno, R. Sofia, W. Moreira, A. Bogliolo, and N. Martins. A User-centric Approach to Trust Management in Wi-Fi Networks. In INFOCOM'2013 Demo/Poster Session, Turin, Italy, April 2013.

[4] L. Lopes, R. Sofia, H. Osman, H. Haci, and H. Zhu. A Proposal for Dynamic Frequency Sharing in Wireless Networks . Journal Paper Under submission, 2014.

[5] H. Osman, H. Haci, H. Zhu, L. Lopes, and R. Sofia. Method and Apparatus for communication in a wireless network. Number EP 13191667.8. (applicant: University of Kent). 